\title{
Genetic Analysis Reveals that Amyloid Precursor Protein and Death Receptor 6 Function in the Same Pathway to Control Axonal Pruning Independent of $\beta$-Secretase
}

\author{
Olav Olsen, ${ }^{1 \star}$ Dara Y. Kallop, ${ }^{2 *}$ Todd McLaughlin, ${ }^{6}$ Sarah Huntwork-Rodriguez, ${ }^{3}$ Zhuhao Wu, ${ }^{1}$ Cynthia D. Duggan, ${ }^{1}$ \\ David J. Simon, ${ }^{1}$ Yanmei Lu, ${ }^{4}$ Courtney Easley-Neal, ${ }^{2}$ Kentaro Takeda, ${ }^{5}$ Philip E. Hass, ${ }^{5}$ Alexander Jaworski, ${ }^{1}$ \\ Dennis D.M. O'Leary, ${ }^{6}$ Robby M Weimer, ${ }^{2,3} \dagger$ and Marc Tessier-Lavigne ${ }^{1} \uparrow$ \\ ${ }^{1}$ Laboratory of Brain Development and Repair, The Rockefeller University, New York, New York 10065, 2Department of Biomedical Imaging, ${ }^{3}$ Department \\ of Neuroscience, ${ }^{4}$ Department of Biochemical and Cellular Pharmacology, and ${ }^{5}$ Department of Protein Chemistry, Genentech Inc., South San Francisco, \\ California 94080, and ${ }^{6}$ Molecular Neurobiology Laboratory, The Salk Institute, La Jolla, California 92037
}

In the developing brain, initial neuronal projections are formed through extensive growth and branching of developing axons, but many branches are later pruned to sculpt the mature pattern of connections. Despite its widespread occurrence, the mechanisms controlling pruning remain incompletely characterized. Based on pharmacological and biochemical analysis in vitro and initial genetic analysis in vivo, prior studies implicated a pathway involving binding of the Amyloid Precursor Protein (APP) to Death Receptor 6 (DR6) and activation of a downstream caspase cascade in axonal pruning. Here, we further test their involvement in pruning in vivo and their mechanism of action through extensive genetic and biochemical analysis. Genetic deletion of DR6 was previously shown to impair pruning of retinal axons in vivo. We show that genetic deletion of APP similarly impairs pruning of retinal axons in vivo and provide evidence that APP and DR6 act cell autonomously and in the same pathway to control pruning. Prior analysis had suggested that $\beta$-secretase cleavage of APP and binding of an N-terminal fragment of APP to DR6 is required for their actions, but further genetic and biochemical analysis reveals that $\beta$-secretase activity is not required and that high-affinity binding to DR6 requires a more $\mathrm{C}$-terminal portion of the APP ectodomain. These results provide direct support for the model that APP and DR6 function cell autonomously and in the same pathway to control pruning in vivo and raise the possibility of alternate mechanisms for how APP and DR6 control pruning.

Key words: degeneration; pruning

\section{Introduction}

Neuronal circuit development involves a generative phase, in which newly born neurons send out axons that branch and innervate synaptic partners, and a regressive phase, in which many branches are pruned back and some neurons die to sculpt the final pattern of connections. Axon pruning can be triggered by pruning inducers such as semaphorins and/or by competition for limiting amounts of trophic factors (Luo and O'Leary, 2005). In

Received Aug. 15, 2013; revised Feb. 24, 2014; accepted March 11, 2014.

Author contributions: 0.0., Y.L., C.E.-N., P.E.H., D.D.M.O., R.M.W., and M.T.-L. designed research; 0.0., D.Y.K. T.M., S.H.-R., Z.W., C.D.D., D.J.S., Y.L., C.E.-N., K.T., A.J., and R.M.W. performed research; 0.0., D.Y.K., T.M., R.M.W., D.D.M.O. and M.T.-L. analyzed data; 0.0., D.D.M.O., R.M.W., and M.T.-L. wrote the paper.

This work was supported by The Rockefeller University, the National Institutes of Health (Grant R01 EY07025 to D.D.M.O.), and Genentech, a wholly owned subsidiary of Roche. D.D.M.O. is partially supported by the Vincent J. Coates Chair in Molecular Neurobiology. We thank members of the Tessier-Lavigne laboratory for discussions and suggestions, Jing Yang for assistance with protein purification, and Karin Sono for help with genotyping.

The authors declare no competing financial interests.

*0.0. and D.Y.K. contributed equally to this work.

tR.M.W. and M.T.-L. are cosenior authors.

Correspondence should be addressed to either of the following: Marc Tessier-Lavigne, Laboratory of Brain Development and Repair, The Rockefeller University, 1230 York Avenue, MS 212, New York, NY 10065, E-mail: marct|@rockefeller.edu; or Robby Weimer, Genentech, Inc., 1 DNA Way, South San Francisco, CA 94080, E-mail: weimer.robby@gene.com.

DOI:10.1523/JNEUROSCI.3522-13.2014

Copyright $\odot 2014$ the authors $\quad 0270-6474 / 14 / 346438-10 \$ 15.00 / 0$ vitro, trophically deprived axons degenerate through activation of a caspase-dependent pathway, which also contributes to pruning in vivo (Nikolaev et al., 2009; Schoenmann et al., 2010; Vohra et al., 2010; Simon et al., 2012), but the mechanisms that lead to caspase activation remain incompletely characterized.

A prior study implicated APP and DR6 (also known as TNFRSF21) in axon degeneration after trophic deprivation (Nikolaev et al., 2009). In vitro, manipulations to interfere with DR6 and APP (using antibodies, siRNA knock down, and, in the case of DR6, genetic inactivation) partially protected axons from degeneration after trophic deprivation and the DR6 ectodomain was shown to bind APP, suggesting that both proteins function in a common pruning pathway. In vivo, genetic deletion of DR6 in mice impaired stereotyped pruning of retinal ganglion cell (RGC) axons (Nikolaev et al., 2009) and pruning of axons of excitatory and inhibitory cortical neurons triggered by sensory deprivation (Marik et al., 2013). These studies are consistent with the involvement of DR6 in pruning in vivo, but both relied on DR6-null mice, so it cannot be excluded that DR6 functioned in the environment rather than degenerating axons themselves. Further, it was not determined whether APP is also required for axon pruning in vivo.

Here, we used RGC axons in vivo as a model to test whether genetic deletion of APP also impairs pruning and shRNA knock 
down to determine whether DR6 and APP function in retinal cells to regulate pruning. We also sought a genetic test of whether APP and DR6 function in the same pathway. Our results are consistent with cell-autonomous involvement of both proteins in the same pathway in pruning in vivo.

Initial biochemical and pharmacological analysis had also suggested a model in which trophic deprivation leads to $\beta$-secretase cleavage of APP, followed by a second cleavage to release an $\mathrm{N}$-terminal fragment of APP, which bound DR6 to trigger degeneration (Nikolaev et al., 2009). We therefore tested the involvement of $\beta$-secretase (BACE1) in pruning in vivo. Unexpectedly, we found that RGC axon pruning was not impaired in Bace1 mutant mice. Further genetic and biochemical analysis in vitro revealed that prior analysis was affected by off-target activities of several pharmacological reagents and supported the conclusion from in vivo analysis that $\beta$-secretase is not required for axon degeneration after trophic deprivation. It also revealed that high-affinity interaction of APP and DR6 requires the more C-terminal E2 domain of APP.

Together, these results support the model that APP and DR6 function cell autonomously and in the same pathway in axon pruning in vivo and raise the possibility of alternate models for the mechanism through which they control degeneration.

\section{Materials and Methods}

Generation of shRNA expression constructs targeting knock down of DR6 and APP and assessment of axon pruning of RGCs labeled in utero. shRNAmediated knock down of DR6 was achieved from a CAGGs-based expression plasmid (Gray et al., 2006) containing the cDNA encoding mCherry (Shaner et al., 2004) and a DR6-targeting sequence (5' AAT CCA AGC AGG AGA AGA GAG GTT TTG GCC ACT GAC TGA CCT CTC TTC CTG CTT GGA TT) embedded into the $3^{\prime}$ UTR flanked by mir155 sequence, which mediates efficient processing of hairpin RNA (Shan et al., 2009). Similarly, shRNA knock down of murine APP was achieved by replacing the DR6 shRNA-related sequence with one targeting APP (5' TTG AGT TTA CCA CAG AAC ATG GTT TTG GCC ACT GAC TGA CCA TGT TCT GGT AAA CTC AA). shRNA-mediated knock down of murine DR6 and APP was confirmed via Western blot analysis using anti-GFP antibody (Aves Labs) or anti-APP antibody Y188 (Abcam) of lysates from HEK cells that coexpressed murine DR6 fused to GFP or murine APP and the corresponding shRNA. To assess axon pruning in vivo, plasmids were introduced into central RGCs by in utero electroporation at embryonic day 13.5 (E13.5; Garcia-Frigola et al., 2007), and axon pruning scored at postnatal day 6 (P6), as described previously (Simon et al., 2012). Error bars in the figures depict SEM.

Sensory neuron cultures. E13.5 dorsal root ganglia (DRG) explants were cultured as described previously (Nikolaev et al., 2009; Simon et al., 2012). For all experiments, DRGs from mutant embryos were compared with DRGs from littermate controls. Briefly, DRGs were plated onto laminin-coated 8-well chamber slides containing Neurobasal/B27, 10 $\mathrm{U} / \mathrm{ml}$ penicillin, $10 \mu \mathrm{g} / \mathrm{ml}$ streptomycin, $292 \mu \mathrm{g} / \mathrm{ml} \mathrm{L}$-glutamine, and 25 $\mathrm{ng} / \mathrm{ml}$ NGF. DRGs were cultured for $7 \mathrm{~d}$ and then deprived of NGF by switching to media lacking NGF and containing $25 \mu \mathrm{g} / \mathrm{ml}$ anti-NGF antibody. Explants were cultured for an additional $34-40 \mathrm{~h}$, during which time the cultures were monitored to assess axonal degeneration and then fixed and stained.

To test for prodegenerative effects of ectodomains or ectodomain fragments in such cultures, at the time of NGF deprivation, $10 \mu \mathrm{g} / \mathrm{ml} \mathrm{sAPP} \beta$ HIS, N-APP-HIS, C-APP-HIS, or DR6-Fc were added. Cultures were examined $30 \mathrm{~h}$ later, before significant degeneration in control cultures had occurred, to determine whether the fragments had accelerated degeneration. Two additional degeneration assays were also used. In the first assay, DRG cultures were established in presence of $50 \mathrm{ng} / \mathrm{ml} \mathrm{NGF}$, but on the second day, the NGF concentration was reduced to $0.5 \mathrm{ng} / \mathrm{ml}$ or $0.1 \mathrm{ng} / \mathrm{ml}$ to make the neurons more vulnerable to insult and proteins were added; cultures were scored $\sim 84 \mathrm{~h}$ later for accelerated degeneration. In the second assay, DRG cultures were established without NGF. In such cultures, $\mathrm{TrkA}^{+}$axons extend robustly but then die progressively over the first $48-72 \mathrm{~h}$ (Z.W., O.O., and M.T.-L., unpublished data). The purified proteins were added at onset of culture and cultures were examined at $26 \mathrm{~h}$ to determine whether there was any acceleration of degeneration. No evidence of enhanced degeneration was observed. All three assays were also repeated with alkaline phosphatase (AP) fusions of the three APP fragments, again without evidence of acceleration of degeneration.

Immunocytochemistry. To assess degeneration, DRG cultures were fixed with $4 \%$ paraformaldehyde in PBS, rinsed in PBS, and then incubated in blocking solution (PBS containing 5\% donkey serum and $0.1 \%$ Triton X-100) for $1 \mathrm{~h}$. Cultures were incubated with anti-Tuj1 antibody (1:1000; Covance) overnight at $4^{\circ} \mathrm{C}$ in blocking solution, then washed in PBS containing $0.1 \%$ Triton $(3 \times, 10 \mathrm{~min})$ and incubated with fluorescently conjugated secondary antibody (Alexa Fluor 568, 1:1000) for $1 \mathrm{~h}$ at room temperature. Slides were coverslipped and visualized using a fluorescence microscope.

Quantification. Axon degeneration was scored using a visual five-point scale and was performed blind to genotype. Because the precise extent of degeneration varied slightly between experiments, degeneration was always normalized within each experiment to the amount of degeneration observed in control DRGs in that experiment.

Binding assays. AP, polyhistidine (HIS), and Fc fusion proteins were produced in HEK293 cells. Cell-binding experiments were performed as described previously (Nikolaev et al., 2009). Briefly, supernatants from 293 cells expressing AP fusions were collected and the concentration of the AP-fusion proteins was determined by measuring AP activity. APproteins were then diluted in AP binding buffer (HBSS, $20 \mathrm{~mm}$ HEPES, $0.2 \% \mathrm{BSA}, 0.1 \% \mathrm{NaN}_{3}, 5 \mathrm{~mm} \mathrm{CaCl}_{2}$, and $1 \mathrm{~mm} \mathrm{MgCl} 2$ ) to $250 \mathrm{~nm}$. Transfected COS cells were then rinsed with AP binding buffer $(2 \times)$ and incubated with diluted AP-proteins for $2 \mathrm{~h}$ on ice. Cells were then rinsed using binding buffer $(5 \times)$ and fixed (3\% paraformaldehyde in PBS) for $12 \mathrm{~min}$ at room temperature. After another series of rinses (HBS $\times 3$ ), endogenous AP activity was quenched by incubating cells in $\mathrm{HBS}$ at $65^{\circ} \mathrm{C}$ for $30 \mathrm{~min}$. Cells were then washed three times in AP buffer containing $100 \mathrm{~mm}$ Tris (9.5), $100 \mathrm{~mm} \mathrm{NaCl}$, abd $50 \mathrm{~mm} \mathrm{MgCl}_{2}$, and AP-protein binding was observed by incubating cells in developing buffer (AP buffer containing 1:50 dilution NBT/BCIP; Roche) overnight at $4^{\circ} \mathrm{C}$.

Proteins used in ELISA assays were purified using standard protocols for affinity purification of HIS-tagged and Fc-tagged proteins. Supernatants from HEK293 cells were incubated with Ni-NTA agarose or protein-A/G Sepharose columns for immobilization of HIS-tagged or Fc-tagged recombinant proteins, respectively. After extensive washes, HIS-tagged and Fc-tagged proteins were eluted from columns using imidazole buffer (200 mM) or low-pH buffer ( $\mathrm{pH} 3.5)$, respectively. Proteins were then further purified by FPLC using a Superose 6 10/300 GL column (GE Healthcare Life Sciences). These purifications yielded proteins of $>95 \%$ purity, as assessed by Coomassie stain. To determine the affinity of binding of the DR6 ectodomain to APP ectodomain fragments, 96well high-binding ELISA plates (R\&D Systems) were coated with $2 \mu \mathrm{g} / \mathrm{ml}$ purified sAPP $\beta$-HIS, C-APP-HIS, or N-APP-HIS in PBS overnight at $4^{\circ} \mathrm{C}$. Wells were then washed with wash buffer (PBS containing $0.05 \%$ Tween 20) three times and then blocked with blocking buffer (PBS containing $1 \% \mathrm{BSA}$ ) for $2 \mathrm{~h}$ at room temperature (RT). Serial dilutions for DR6-Fc were prepared in blocking buffer and incubated in wells overnight at $4^{\circ} \mathrm{C}$. Plates were then washed with washing buffer $(4 \times)$ and incubated with anti-human HRP antibody (1:500) in blocking buffer for $2 \mathrm{~h}$ at RT. After a final series of washes $(4 \times)$, wells were incubated with color reagents (R\&D Systems) until signal was visible. Reaction was stopped by the addition of stop solution and signal was read at $450 \mathrm{~nm}$ in a plate reader (Tecan). Specific binding was determined by subtracting optical density (OD) $450 \mathrm{~nm}$ readings from wells in which DR6-Fc was incubated from readings from wells to which BSA had been adsorbed (again, as a $2 \mu \mathrm{g} / \mathrm{ml}$ solution). Binding curves were generated by normalizing OD 450 absorbance readings to the greatest signal for each adsorbed protein in a given experiment. Normalized readings were then averaged across experiments.

For immunoprecipitations, Fc proteins from HEK cell supernatants immobilized to protein A/G beads were washed with PBS $(3 \times)$ and 
incubated in PBS at $4^{\circ} \mathrm{C}$ overnight with HIStagged proteins purified by Ni-NTA columns. Beads were washed with PBS $(5 \times)$ and proteins were eluted in protein sample buffer. Immunoprecipitated complexes were separated by SDS-PAGE and transferred to PVDF membranes for analysis by Western blot. Anti-HIS antibody (1:1000) was incubated in TBS containing $0.1 \%$ Tween 20 and $1 \%$ BSA overnight at $4^{\circ} \mathrm{C}$. HRP-conjugated secondary antibodies (Jackson ImmunoResearch Laboratories) were used at 1:10,000 and detected by ECL.

\section{Results}

\section{APP regulates $\mathrm{RGC}$ axon pruning} in vivo

Previously, we showed that DR6 contributes to refining axon projections in the superior colliculus (SC) in vivo. Initially, temporal RGC axons extend into the posterior superior colliculus (PSC), overshooting their final target, the termination zone (TZ) in the anterior SC. By P7, however, exuberant RGC axons in the SC have been refined to the TZ through a selective axon degeneration pruning process (Luo and O'Leary, 2005). Using DiI tracing of axons, we showed previously that this process is impaired in DR6 knock-out $(\mathrm{KO})$ mice, resulting in a significant number of RGC axons and arbors that persist beyond the $\mathrm{TZ}$ at $\mathrm{P} 6$, when pruning is almost complete in controls (Nikolaev et al., 2009).

To determine whether APP plays a similar role in RGC axon pruning in vivo, we performed focal DiI injections limited to the periphery of the temporal retina of P5 APP KO and wild-type littermates and analyzed the retinocollicular projections in the SC at P6. As seen previously, in wild-type mice, DiI-labeled axons show a restricted localization in the $\mathrm{TZ}$ at that age, reflecting almost complete pruning (Fig. 1). In contrast, APP KO animals, like DR6 KOs (Nikolaev et al., 2009) and Caspase3 KOs (Simon et al., 2012), show a greater number of DiI-labeled RGC axons that persist beyond the TZ at P6 compared with control (Fig. 1). This difference was significant, as assessed by quantification of area coverage by DiI-labeled axons in the SC (Fig. 1), and appeared to be as great or greater than that observed previously in DR6 or Caspase3 KO mice (Nikolaev et al., 2009; Simon et al., 2012).

Therefore, like DR6, APP regulates pruning of the retinocollicular projection in vivo. Because protection is only partial, there must be additional mechanisms that collaborate with APP to mediate degeneration; one candidate is the close APP relative APLP2, especially because it appears to function redundantly with APP in controlling neuromuscular junction formation (Wang et al., 2005; see Discussion).

\section{APP and DR6 function cell autonomously in retinal cells to promote axon pruning}

APP could function in RGCs or in the SC to regulate pruning. To address whether it functions in retinal cells, we used an shRNA directed against APP to knock down expression of APP in retinal cells in wild-type mice. To deliver the shRNA, we used in utero electroporation into the retina. We previously used electroporation of a GFP expression plasmid into the retina to show that retinocollicular pruning of central retinal RGC populations is impaired in Caspase- 3 and Caspase- $6 \mathrm{KO}$ mice by counting the number of $\mathrm{GFP}^{+}$axons in the PSC (Simon et al., 2012).

In control experiments, an shRNA directed against APP (shAPP) effectively knocked down APP expression in transfected cells, whereas a control plasmid targeting LacZ (shLacZ) had no effect (Fig. 2A). Using wild-type E13.5 embryos, the GFP expression plasmid was then coelectroporated in utero with shLacZ or shAPP. The shRNA targeting APP resulted in a significant increase in the number of unpruned GFP-expressing axons persisting beyond the TZ compared with shLacZ (Fig. $2 B-D$ ). This difference did not result from differences in TZ size between the wild-type and shAPP groups, because normalizing against these variables did not account for it (Fig. 2D). These data indicate that APP expression in retinal neurons is necessary for normal refinement of RGCs axons in the superior colliculus by P6.

To examine whether DR6 also functions cell autonomously in pruning, we next performed shRNA knock down of DR6 in retinal cells using an shRNA against DR6 (shDR6), which, in control experiments, effectively knocked down DR6 expression in transfected cells (Fig. 3A). As observed for electroporation with shAPP, coelectroporation of shDR6 along with the GFP expression plasmid into the retina was sufficient to impair axon pruning in the $\mathrm{SC}$, as determined by a greater number of $\mathrm{GFP}^{+}$axons extending into the anterior superior colliculus compared with RGCs electroporated with the nontargeting shLacZ control (Fig. $3 B-D)$. Again, this difference did not result from differences in TZ size between the wild-type and DR6 KO groups (Fig. 3B). Together, these data support a model in which DR6 and APP 
A
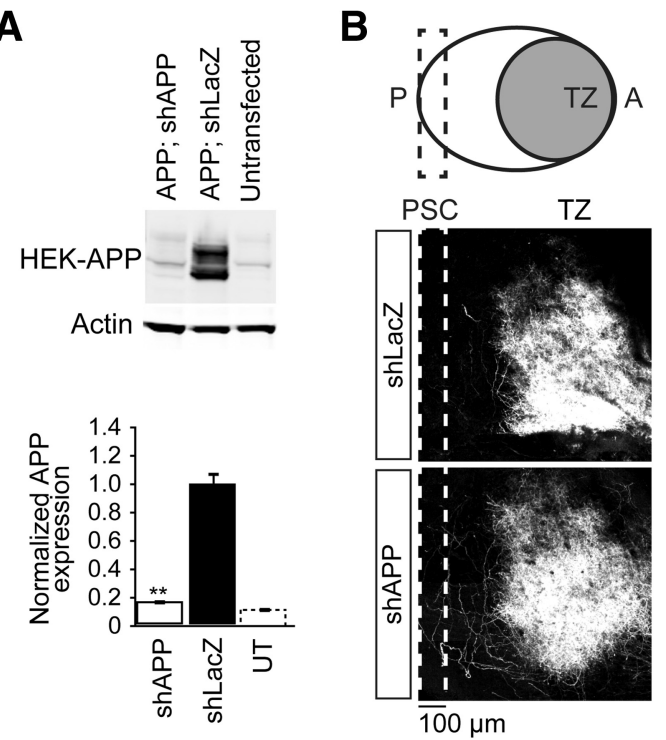

D

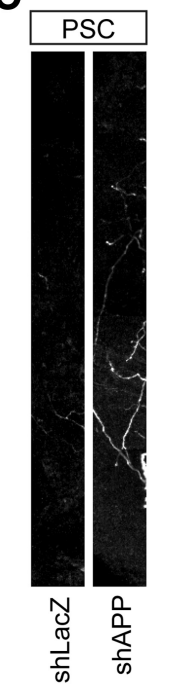

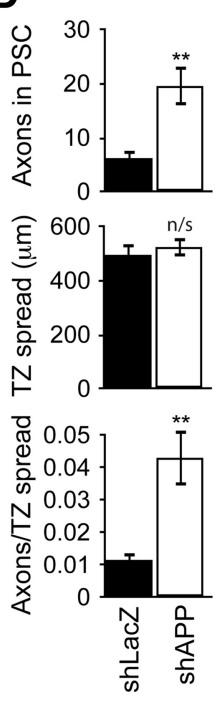

Figure 2. $\quad$ shRNA knock down of APP in retina impairs pruning of RGC axons in the SC. $A$, Representative Western blot (top) and quantification (bottom) of APP and actin protein from HEK cells cotransfected with an APP-expressing plasmid and an shRNA plasmid against $A P P$ or $L a c Z$ or untransfected (UT), as indicated, $n=3$ repeat experiments. $B$, The right eyes of E13.5 embryos were co-electroporated with GFP expression plasmid and shRNA against LacZ or APP and RGC axon projections in the $S C$ were examined at P6. At P1, all axons labeled in this way project to the posterior (P) end of the SC (i.e., the PSC; Simon et al., 2012; Fig. 7 A,B). As shown in the diagram (top), by P8, axons have pruned back so that they now only occupy the TZ. The next two panels show representative maximum intensity projection images of GFP-positive axons within the SC at P6 in WT mice coexpressing GFP and shRNA against LacZ (shLacZ) or APP (shAPP). Note that by P6 the pruning of axons in the PSC in shLacZ is largely complete. $C$, Representative images of the PSC in WT mice expressing shRNA against LacZ or APP. D, Quantification of the number of GFP-positive axons in the PSC, the spread of GFP labeling along the anterior-posterior axis of the SC as an estimate of the size of the $\mathrm{TZ}$, and the number of GFP-positive axons in the PSC normalized to TZ size in mice expressing shLacZ or shAPP. ${ }^{* *} p<0.01 ; \mathrm{n} / \mathrm{s}$, not significant $(p>0.1)$.

A
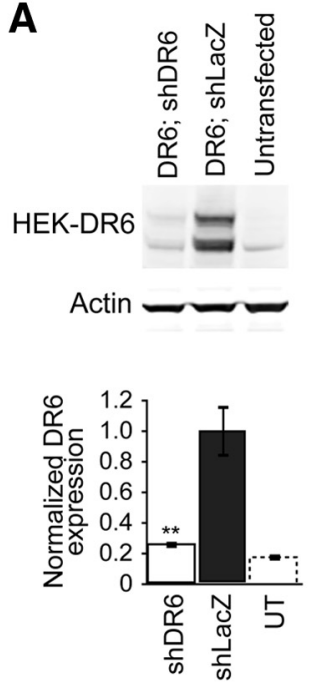

B

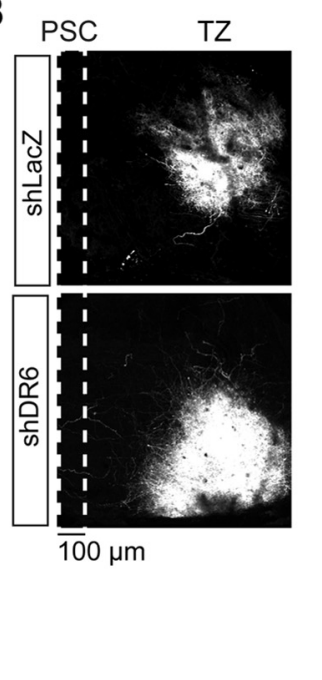

C

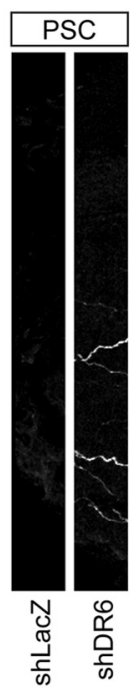

D

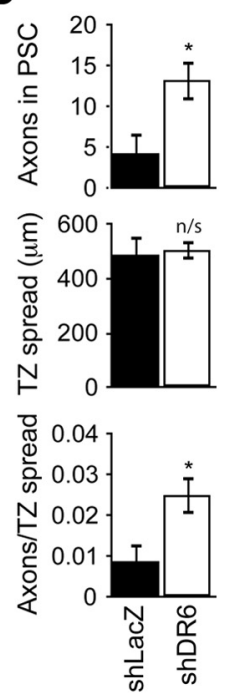

Figure 3. shRNA knock down of DR6 in the retina impairs pruning of RGC axons in the SC. $A$, Representative Western blot (top) and quantification (bottom) of DR6 and actin protein from HEK cells cotransfected with a DR6-expressing plasmid and an shRNA plasmid against DR6 or LaCZ, or untransfected (UT), as indicated. $n=3$ repeat experiments. ${ }^{* *} p<0.01$. $\boldsymbol{B}, \boldsymbol{C}, \mathrm{RGCS}$ of E13.5 embryos were coelectroporated in utero with GFP expression plasmid and shRNA against LacZ or DR6. $\boldsymbol{B}$, Representative maximum intensity projection images of GFP-positive axons within the SC at P6 in WT mice coexpressing GFP and shRNA against LacZ or DR6. C, Representative images of the PSC in WT mice expressing shRNA against LacZ or DR6. D, Quantification of the number of GFP-positive axons in the PSC, the spread of GFP labeling along the anterior-posterior axis of the SC as an estimate of the size of the $\mathrm{TZ}$, and the number of GFP-positive axons in the PSC normalized to TZ size in mice expressing shLacZ. ${ }^{*} p<0.05 ; \mathrm{n} / \mathrm{s}$, not significant $(p>0.1)$. both act cell autonomously in the refinement of RGC axon projections in the SC.

\section{Nonadditivity of effects of loss of DR6 and APP function}

The similarity in phenotypes observed with $A P P$ and $D R 6$ knock down or knockout is consistent with APP and DR6 functioning in the same pathway, but does not prove it. A more stringent test is to examine whether simultaneous loss of both APP and DR6 function gives additive or nonadditive effects on pruning. Nonadditivity would support the model that they function in the same pathway. Additivity could still be compatible with them being in the same pathway (if, for example, there are other factors that act redundantly with both of them, such as close family members), but it could also be compatible with them being in distinct, parallel pathways. To test the effects of combined loss of function, we performed shRNA knock down of APP in a DR6 mutant background; as a control, we introduced shLacZ into the DR6 mutant. Axonal pruning was impaired to the same extent whether shAPP or shLacZ was introduced into the DR6 mutant background (Fig. 4). Therefore, APP knock down on top of DR6 knock-out does not augment the effect of DR6 knock-out alone. This nonadditivity of loss-of-function effects supports the model that APP and DR6 function in the same pathway.

\section{RGC pruning does not require BACE-1} Pharmacological studies suggested that APP and DR6 function in pruning of sensory axons in vitro requires cleavage of APP by $\beta$-secretase (BACE- 1 ; Nikolaev et al., 2009). To test the involvement of BACE-1 in retinocollicular pruning in vivo, we studied pruning of the retinocollicular projection in Bace-1 knock-out mice, again by electroporating a GFPencoding plasmid in the retina in utero (Fig. 5). Unexpectedly, pruning of the retinocollicular projection was not impaired in the Bace-1 mutant: by P6, the extent of pruning in the Bace-1 knock-out was not decreased compared with that observed in wild-type animals and was in fact slightly greater (Fig. 5). This argues against a necessary role for cleavage of APP by BACE- 1 in pruning and prompted us to revisit the involvement of $\beta$-secretase in the degeneration of sensory axons in vitro.

\section{APP and DR6 in sensory \\ axon degeneration}

The involvement of DR6 and APP in axon pruning in vitro was described in 
A

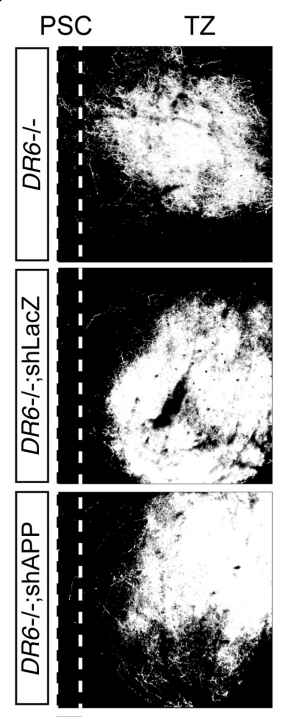

$\overline{100} \mu \mathrm{m}$
B

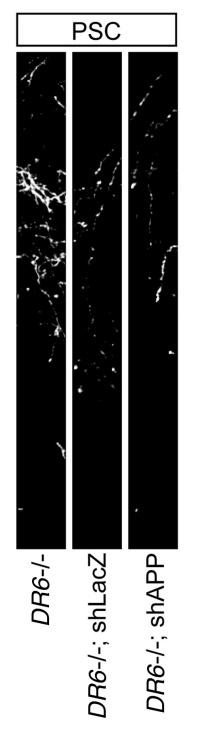

C

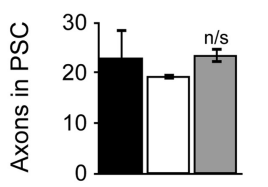

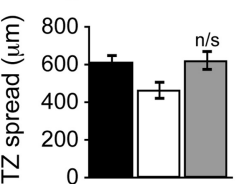

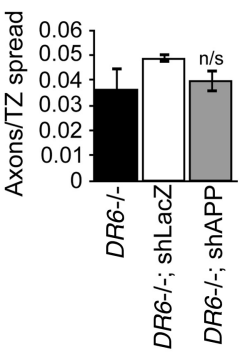

Figure 4. shRNA knock down of $A P P$ in the retina of $D R 6$ knock-out animals does not impair pruning of RGC axons in the SC beyond what is observed in the DR6 knock-out alone. RGCs ofE13 DR6 KO embryos were coelectroporated in utero with GFP expression plasmid and shRNA against LacZ or APP. A, Representative maximum intensity projection images of GFP-positive axons within the SC at P6 in DR6 K0 mice coexpressing GFP alone or an shRNA against LacZ or $D R 6 . B$, Representative images of the PSC in DR6 KO mice expressing GFP alone or shRNA against LacZ or APP. C, Quantification of the number of GFP-positive axons in the PSC, the spread of GFP labeling along the anterior-posterior axis of the SC as an estimate of the size of the TZ, and the number of GFP-positive axons in the PSC normalized to TZ size in mice expressing shLacZ. n/s, not significant $(p>0.05)$.

A

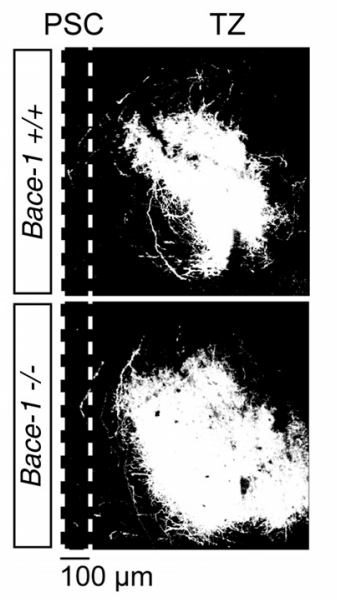

B

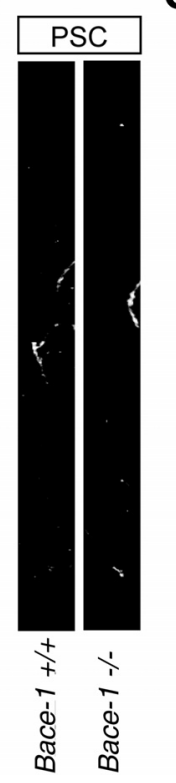

C
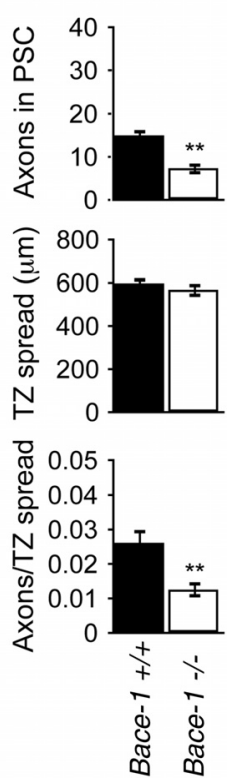

Figure 5. Pruning of RGC axons in the absence of BACE-1. $\boldsymbol{A}$, Representative maximum intensity images of the SC from P6 WT and Bace- $1 \mathrm{KO}$ mice that were electroporated with a GFP expression plasmid at E13. Bace-1 K0 mice exhibit an appropriately localized and sized TZ and no distinguishable difference in the degree of RGC axon pruning compared with wild-type littermates. $\boldsymbol{B}$, Representative images of the PSC in WT and Bace-1 KO mice are shown. $\boldsymbol{C}_{r}$ Quantification of the number of GFP-positive axons that persist in the PSC, the anterior-posterior spread of GFP labeling in the TZ, and the number of GFP-positive axons in the PSC normalized to the size of the TZ in WT and Bace- $1 \mathrm{KO}$ mice. ${ }^{* *} p<0.01$; n/s, not significant $(p>0.1)$.

the context of a model of sensory axon degeneration in response to trophic factor deprivation (Nikolaev et al., 2009). E13.5 mouse embryonic sensory ganglia are cultured with NGF to elicit outgrowth of TrkA ${ }^{+}$sensory axons for either $2 \mathrm{~d}$ (shortterm cultures) or $7 \mathrm{~d}$ (long-term cultures), and then deprived of NGF (by its removal and the addition of a function-blocking antibody). This results in degeneration of axons in $\sim 14-18 \mathrm{~h}$ in short-term cultures and $\sim 36-40 \mathrm{~h}$ in long-term cultures; the slower degeneration in long-term cultures reflects the fact that these neurons become progressively less trophic factor dependent as they age (Simon et al., 2012). Degeneration in both cases is caspase dependent and can be assessed by tubulin fragmentation, as visualized by staining for $\beta$ III tubulin (Tuj1; Simon et al., 2012). In what follows, we focus on the long-term (7 d) cultures, because the slower rate of degeneration makes it possible to test for even small protective effects.

To provide a baseline for genetic analysis of $\beta$-secretase activity in vitro, we performed genetic analysis of $D R 6$ and $A P P$ knockout neurons in culture. For both DR6 and APP knock-outs, sensory neurons from E13.5 mutant and wild-type littermates were cultured for $7 \mathrm{~d}$ with NGF and then deprived of it. Genetic deletion of either $D R 6$ or APP partially protects against degeneration of sensory axons in culture (Fig. $6 A-D$ ). In each case, the protective effect was significant but modest, for example, when compared with the effects of Caspase-3 knock-out (Simon et al., 2012). However, it should be noted that the five-point degeneration index used for quantification leads to a compression of values, so that the magnitude of protection is not as well captured by the quantification (Fig. $6 C, D$ ) as it is by visual inspection (Fig. $6 A, B)$. The fact that protection is only partial indicates the operation of additional mechanisms that regulate degeneration in these axons, consistent with the partial protection of RGC axons in vivo in the $A P P$ and $D R 6$ knock-outs.

Interestingly, protective effects were not as great as reported in studies with function-blocking antibodies: monoclonal antibody 3F4 to DR6 (anti-DR6.1) and polyclonal antibody anti-N-APP (Thermo Fisher) to the N-terminal portion of APP, which were used previously (Nikolaev et al., 2009), protect axons to a greater extent than do genetic deletion of $D R 6$ or APP (Fig. 6C-E). In the prior study (Nikolaev et al., 2009), it was observed in a head-tohead comparison in compartmented (Campenot) chambers that DR6 knock-out protected axons to a similar extent as anti-DR6.1, but in the more extensive analysis here, we observed systematically greater protection with the antibody. Importantly-and unexpectedly-we found that protection afforded by anti-DR6.1 persisted in DR6 knock-out cultures and protection from anti-NAPP persisted in APP knock-out cultures (Fig. $6 C-E$ ), indicating that strong protection by the antibodies results from off-target actions. At least for anti-N-APP, this might relate to the recent report that multiple anti-APP antibodies label axons nonspecifically; that is, that labeling by immunohistochemistry is maintained even when APP is genetically deleted (Guo et al., 2012), which we found is also true for the anti-N-APP antibody used here (data not shown). Moreover, one batch of control IgG (IgG2) also provided a modest but significant protective effect, whereas another (IgG1) did not protect (Fig. 6E). Further studies are necessary to determine the nature of these off-target effects, including whether engagement of Fc receptors on axons plays a role (which could explain the action of IgG2). Meanwhile, these results suggest caution when interpreting the effects of antibodies in this assay.

We next examined the involvement of $\beta$-secretase in sensory axon degeneration. Previously, a requirement for $\beta$-secretase in 

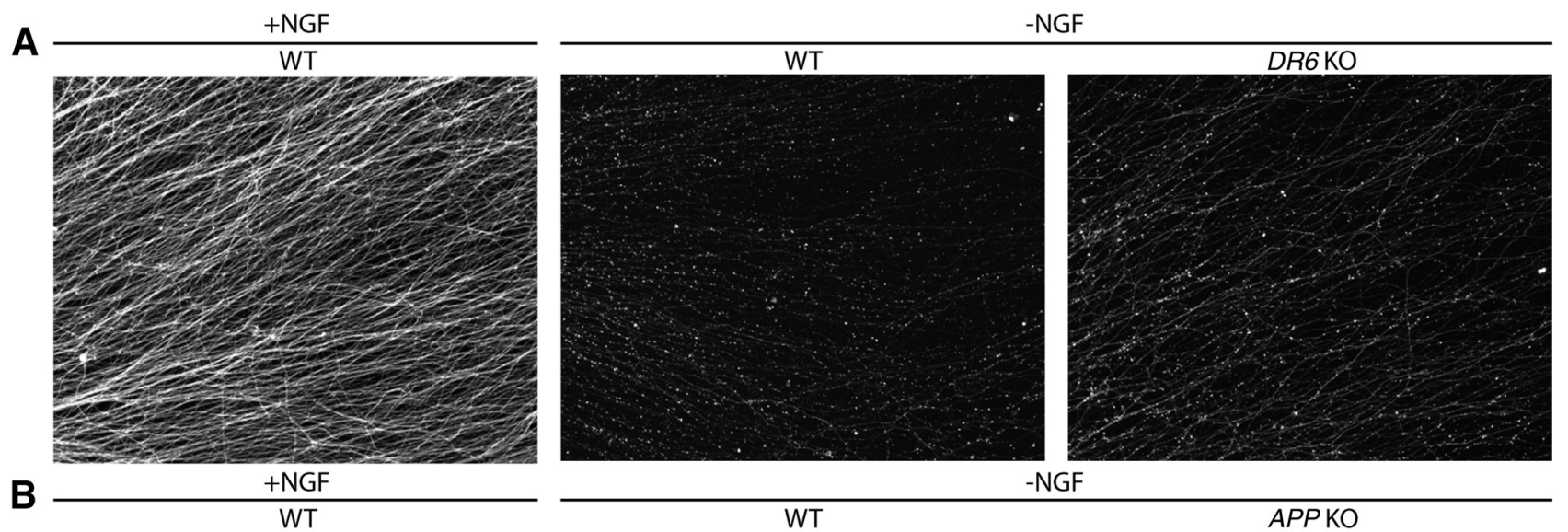

B

WT

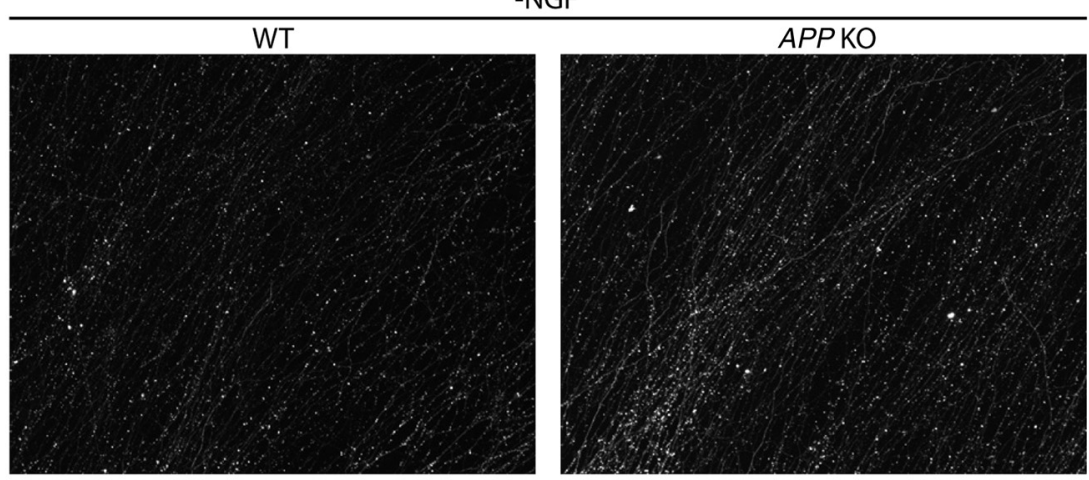

C

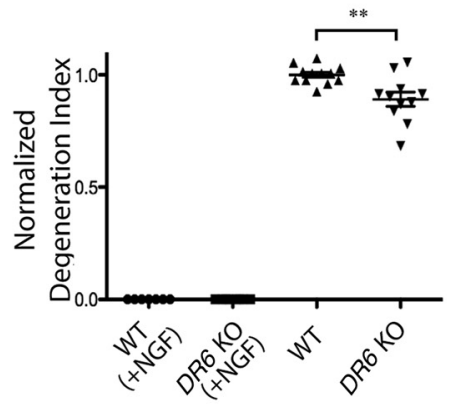

D

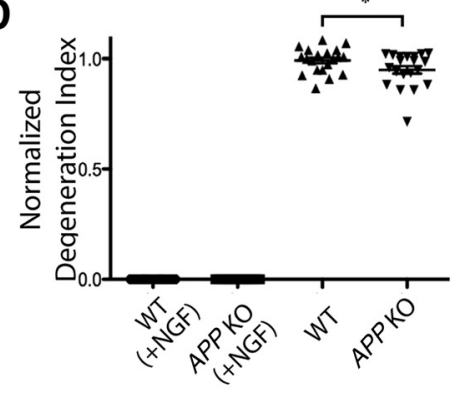

E

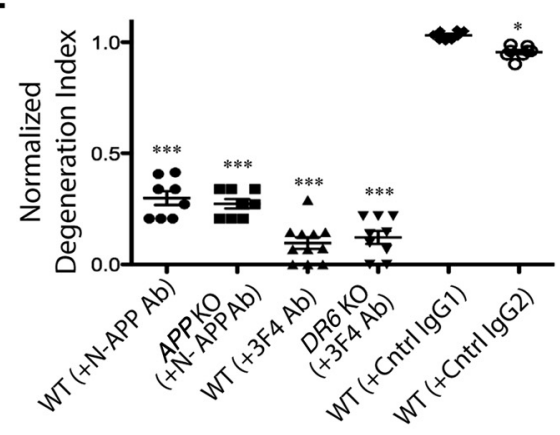

$\mathbf{F}$

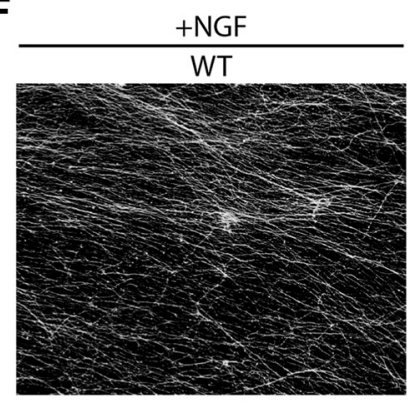

$-\mathrm{NGF}$

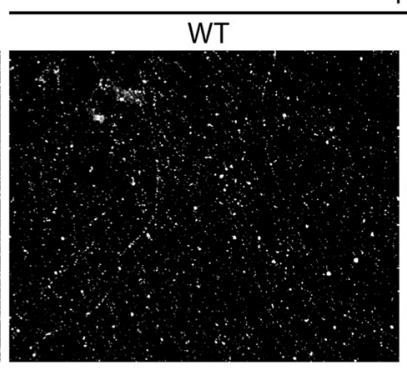

G

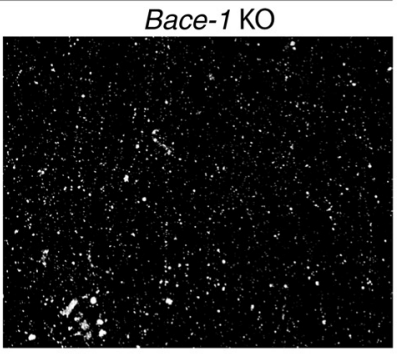

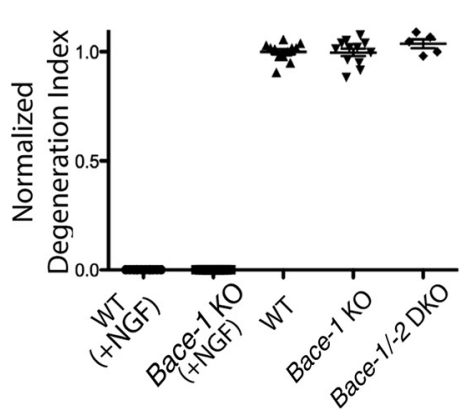

Figure 6. Genetic deletion of DR6 or APP, but not Bace-1, partially protects sensory axons from degeneration after NGF withdrawal in vitro. $A, B, D R G s$ from E13.5 DR6 (A) or APP (B) K0 embryos and wild-type (WT) littermates were cultured for $7 \mathrm{~d}$ in the presence of NGF ( $25 \mathrm{ng} / \mathrm{ml}$; +NGF) and then deprived of NGF by exchanging culture media containing NGF with media lacking NGF and containing a function-blocking anti-NGF antibody ( $25 \mu \mathrm{g} / \mathrm{ml}$; - NGF). After $36 \mathrm{~h}$ of NGF deprivation, cultures were fixed and axon degeneration was visualized by fluorescence microscopy using an antibody against $\beta \mathrm{III}$-tubulin (Tuj1). Representative images are shown for WT and K0 axons cultured under various conditions. $C, D$, Axon degeneration was scored using a five-point scale and then normalized to the degeneration scored for WT axons under -NGF conditions. DRGs from DR6 $(n=11 ; \boldsymbol{C})$ and $A P P(n=20 ; \boldsymbol{D})$ K0 embryos showed partial protection from axon degeneration compared with WT littermates. $\boldsymbol{E}$, To test the specificity of protection against axon degeneration offered by the anti-DR6 and anti-APP antibodies, antibodies were included in media at the time of NGF deprivation. Antibodies were used at $20 \mu \mathrm{g} / \mathrm{ml}$. The anti-DR6 (3F4) and anti-N-APP antibodies prevented axon degeneration following NGF withdrawal to a greater extent than in the knock-outs, but this enhanced protection was still observed when using DRGs from the respective DR6 or APPKO embryos. One control antibody (lgG1, $20 \mu \mathrm{g} / \mathrm{ml})$ had no effect on axon degeneration $(n=3)$, but a second ( $(\mathrm{gG} 2,20 \mu \mathrm{g} / \mathrm{ml})$ showed significant, though modest, protection $(n=4) . F$, DRGs from E13.5 Bace- $1 \mathrm{KO}$ embryos and WT littermates were cultured for $7 \mathrm{~d}$ in presence of NGF $(25 \mathrm{ng} / \mathrm{ml} ;+$ NGF) and then deprived of NGF by exchanging culture media containing NGF with media lacking NGF and containing a function blocking anti-NGF antibody (25 $\mu \mathrm{g} / \mathrm{ml} ;-\mathrm{NGF).} \mathrm{G,} \mathrm{DRGs}$ from Bace-1 $(n=12)$ and Bace-1/2 KO embryos $(n=5)$ do not show any protection from degeneration. Error bars indicate SE. ${ }^{*} p<0.05 ;{ }^{* *} p<0.01$; ${ }^{* * *} p<0.001 ; n / s$, nonsignificant $(p>0.05)$. 
this process was suggested by the finding that three structurally distinct inhibitors of BACE-1 (OM-99, BACE inhibitor IV, and AZ29) protect axons against degeneration in response to NGF deprivation and that the $\mathrm{IC}_{50}$ for protection by AZ29 was comparable to its $K_{\mathrm{d}}$ for inhibition of BACE-1 (Nikolaev et al., 2009). To test involvement of BACE-1 genetically, we examined axon degeneration of sensory axons after NGF deprivation in longterm cultures from Bace-1 KO animals and compared them with those seen in cultures from wild-type mice. No significant difference in degeneration was observed (Fig. $6 F, G$ ). Although APP is not thought to be a substrate for BACE-2 (the other member of the $\beta$-secretase family), the pharmacological inhibitors also inhibit BACE-2 because of the similarity of BACE active sites. We therefore studied sensory neurons from Bace-1;Bace-2 double $\mathrm{KO}$ mice, but found that their axons degenerate in the same way as those from control littermates (Fig. 6G). We confirmed that AZ29 protected axons from degeneration after NGF deprivation in wild-type sensory neurons, as described previously (Nikolaev et al., 2009), but observed a similar protective effect of AZ29 on sensory axons from Bace-1;Bace-2 double KO mice (mean degeneration index in the presence of AZ29: wild-type axons, $0.73 \pm$ 0.4 ; Bace-1;Bace- $2 \mathrm{KO}$ axons, $0.76 \pm 0.5 ; p>0.1, n=5$ experiments). Therefore, AZ29 produces its effect in an off-target manner, which is surprising given the dose dependence of its protective effect and its reported specificity and given that protection by small-molecule inhibitors is not commonly observed in this assay (Chen et al., 2012). Collectively, these results also indicate that BACE-1 (and BACE-2) is not required for degeneration of sensory axons in response to trophic factor deprivation, which is consistent with the fact that Bace-1 is not required for RGC pruning in vivo.

\section{APP-DR6 binding}

The generation of an N-terminal fragment of APP after trophic deprivation in sensory axons (Nikolaev et al., 2009) and sympathetic axons (Vohra et al., 2010) was proposed to be dependent on $\beta$-secretase (Nikolaev et al., 2009). Given the evidence that $\beta$-secretase activity is not required for degeneration, we revisited the biochemical (binding) and functional (prodegenerative) effects of N-APP. The prior study (Nikolaev et al., 2009) had used N-APP from two sources, commercial (Thermo Fisher) and inhouse (Genentech), which gave consistent results. However, both preparations were only partially purified and biochemical analyses revealed them to contain contaminants and aggregated material (data not shown). To guard against nonspecific effects, we set out to obtain purer and nonaggregated N-APP. As purification proceeded, we unexpectedly found that the prodegenerative effects of N-APP were lost, as was binding to the DR6 ectodomain (fused to alkaline phosphatase: DR6-AP) observed by ELISA (Fig. $7 E)$. One possibility is that the binding and functional effects seen with earlier material were caused by aggregates that may have accumulated during partial purification; an alternative is that a contaminant in the partially purified material contributed to activity and/or binding.

Because we consistently observe robust and reproducible binding of DR6-AP to COS-7 cells expressing full-length APP (Nikolaev et al., 2009; Fig. 7), the lack of binding of more purified $\mathrm{N}$-APP to DR6-AP suggested that the N-terminal portion of APP might not be the major mediator of the interaction. We therefore performed a more extensive structure-function analysis. COS-7 cells were transfected with expression plasmids for full-length DR6 (COS-DR6), APP (COS-APP), or the axon guidance receptor DCC as a control (COS-DCC). Cells were incubated with AP fusions to the ectodomain of DR6 (DR6-AP), the sAPP $\beta$ fragment (depicted in Fig. $7 A$ ) of the APP ectodomain (sAPP $\beta$-AP), or, again as a control, the ectodomain of DCC (DCC-AP). As previously reported, we observed specific binding of DR6-AP to COS cells expressing APP, but not to cells expressing DR6 itself or DCC; in the reverse configuration, we also consistently observed specific binding of sAPP $\beta$-AP to COS cells expressing DR6, but not APP or DCC (Fig. $7 B$ ). In independent experiments, similar selective binding was also observed if DR6 or APP was expressed in HEK cells (data not shown). To further delineate the region of sAPP $\beta$ that mediates binding to DR6, we created AP fusions to $\mathrm{N}$-APP (N-APP-AP), to the remainder of the APP ectodomain (usually referred to as sAPP-E2), and, within sAPP-E2, to a smaller fragment lacking the linker region (sometimes referred to as C-APP; for details of domains, see Fig. 7A). We observed binding of sAPP-E2-AP and C-APP-AP to COS cells expressing DR6 (Fig. 7C), whereas binding was not detected with N-APP-AP in this assay (Fig. $7 C$ ), consistent with the results using more purified N-APP. These results indicate that a more C-terminal portion of the APP ectodomain mediates binding of APP and DR6 ectodomains.

We next examined whether these binding interactions observed in a cellular context could also be detected using purified proteins. Soluble forms of sAPP $\beta, \mathrm{N}$-APP, and C-APP tagged with a HIS sequence, and soluble DR6-Fc and DCC-Fc (as a control) were produced and purified to apparent homogeneity (see Materials and Methods). Purified DR6-Fc or DCC-Fc was incubated with each of the three purified APP ectodomain fragments and complexes were pulled down using Protein A/G beads. Selective coimmunoprecipitation with DR6-Fc was observed only for sAPP $\beta$-HIS or C-APP-HIS (Fig. 7D), which is consistent with the results of the cell-binding experiments. To obtain a quantitative estimate of the strength of interactions, we used an ELISA assay in which the purified APP ectodomain fragments were individually adsorbed to the substrate, and then incubated with purified DR6-Fc. Again, high-affinity binding of DR6-Fc was readily detected to immobilized sAPP $\beta$-HIS $\left(\mathrm{EC}_{50}=1.4 \mathrm{nM}\right)$ and C-APP-HIS ( $\mathrm{EC}_{50}=10.1 \mathrm{~nm}$ ) but not to N-APP-HIS (Fig. $7 E$ ). The lower affinity of binding of DR6-Fc to C-APP than to sAPP $\beta$ could reflect involvement of other, more $\mathrm{N}$-terminal, portions of the APP ectodomain beyond C-APP in binding; alternatively, it could reflect greater steric hindrance when C-APP is adsorbed to the solid substrate compared with the longer sAPP $\beta$ fragment.

We next examined whether any of these purified soluble APP ectodomain fragments could elicit degeneration. DRG neurons were cultured for $7 \mathrm{~d}$ and then deprived of NGF. At that time, purified sAPP $\beta$-HIS, C-APP-HIS, N-APP-HIS, and DR6-Fc proteins $(10 \mu \mathrm{g} / \mathrm{ml})$ were applied and cultures were examined $30 \mathrm{~h}$ later, when control cultures were just beginning to degenerate. No acceleration of degeneration was observed, as assessed visually and by quantification of the degeneration index (ratio compared with control cultures: $1.0 \pm 0.1,1.02 \pm 0.08,0.96 \pm 0.11$, and $1.02 \pm 0.09$ for sAPP $\beta$-HIS, C-APP-HIS, N-APP-HIS, and DR6-Fc, respectively; not significant, $p>0.1$ ), extending the finding of lack of effect of fully purified N-APP. Two other degeneration paradigms were also tested (see Materials and Methods), again without detectable effects on degeneration (data not shown). It is possible that degenerative effects would require higher-order clustering of the soluble constructs, as observed for other membrane-bound ligand-receptor systems, but these results also open the possibility of alternate models for how APP and DR6 control pruning (see Discussion). 


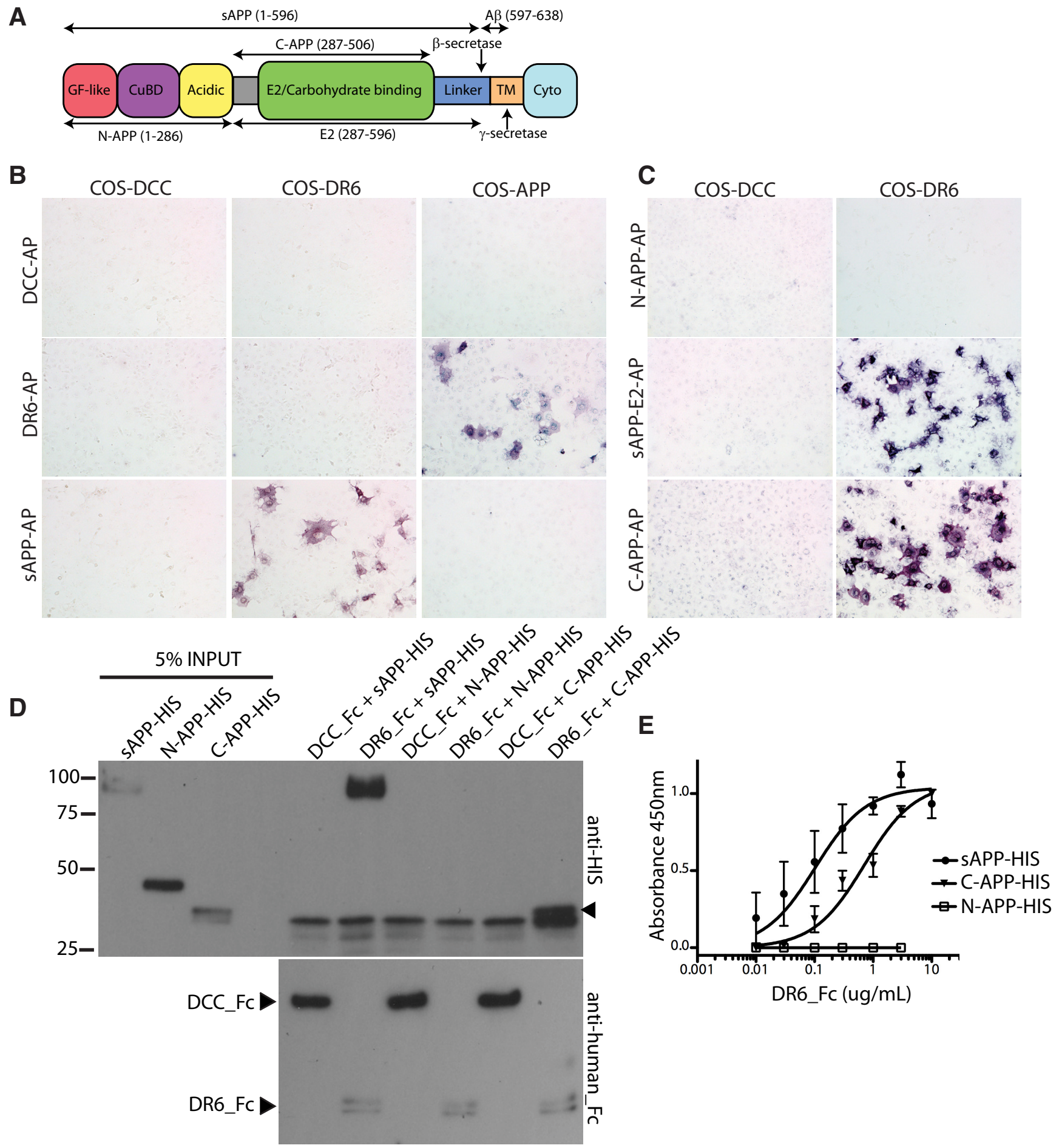

Figure 7. The C-APP domain of APP is required for high-affinity interaction of the APP ectodomain with DR6. A, Schematic diagram of the domain structure of APP, and nomenclature used for AP-fusion proteins. GF-like, Growth factor-like domain; CuBD, copper binding domain; TM, transmembrane domain; Cyto, cytoplasmic domain. Note that the APP ectodomain fragments studies here (SAPP $\beta, N$-APP, SAPP-E2, and C-APP) do not include the A $\beta$ sequence. B, COS-7 cells were transfected with constructs for DCC (control) or full-length DR6 or APP. Two days after transfection, cells were incubated with DCC-AP (control), DR6-AP (aas: 1-349), or sAPP $\beta$-AP (aa 1-596) fusion proteins and tested for binding using an AP substrate. Control (DCC-transfected) cells showed negligible background AP activity and no binding for any of the AP fusions proteins. COS-7 cells expressing DR6 showed a specific interaction with SAPP $\beta$-AP, but not with either DCC-AP or DR6-AP proteins. Further, COS-7 cells expressing APP showed a specific interaction with DR6-AP, but not with either of the DCC-AP or SAPP $\beta$-AP proteins. C, To delineate the minimal region of APP that mediates interaction with DR6, COS-7 cells expressing DCC (control) or DR6 were incubated with N-APP-AP (aa 1-286), sAPP-E2-AP (aa 287-596), or C-APP-AP (aa 287-506) fusion proteins. Only sAPP-E2-AP and C-APP-AP showed specific binding to DR6-expressing cells, whereas no detectable N-APP-AP binding was observed. D, Direct interaction between the ectodomains of DR6 and APP was determined by immunoprecipitation. DR6-Fc and DCC- Fc fusion proteins from HEK293 supernatants were immobilized on protein A/G agarose beads and incubated with HIS-tagged sAPP $\beta$, C-APP, or N-APP proteins. DR6-FC, but not DCC-FC, selectively immunoprecipitated both SAPP $\beta$-HIS and C-APP-HIS, but not N-APP-HIS. E, The affinity of the interaction of DR6 and APP was assessed by ELISA. Consistent with the cell-binding and immunoprecipitation data, DR6-Fc shows a specific and high-affinity interaction with wells coated with sAPP-HIS (EC $50=1.4 \mathrm{nM}$ ) or C-APP-HIS (EC ${ }_{50}=$ $10.1 \mathrm{~nm}$ ) proteins, but not N-APP-HIS-coated wells. 


\section{Discussion}

In this study, we set out to test the involvement of APP and DR6 in axonal pruning in vivo using stereotyped pruning of the retinocollicular projection as a model. Our results directly support the idea that APP and DR6 contribute cell autonomously and in the same pathway to axon pruning in vivo. However, we also found that BACE1 is not required for RGC pruning in vivo, leading us to revisit the more detailed model for how APP and DR6 function in pruning. Further genetic and biochemical analysis in vitro revealed that prior in vitro analysis was affected by off-target action of several pharmacological reagents and supports the conclusion from in vivo analysis that $\beta$-secretase is not required for pruning. It also raises the possibility of alternate models for APP and DR6 involvement in pruning.

\section{Regulation of pruning in vivo by APP and DR6}

Pruning of the retinocollicular projection is a useful model for analysis of developmental axonal degeneration in vivo because it is protracted and stereotyped. The pruning occurs between P2 and $\mathrm{P} 7$, so analysis at $\mathrm{P} 6$ can readily reveal impairments in pruning. In this way, we have shown that genetic deletion of DR6 impairs pruning (Nikolaev et al., 2009), as does genetic deletion of Caspase- 3 or Caspase-6 (Simon et al., 2012). We extend those studies by showing here that genetic deletion of $A P P$ also partially protects against degeneration. The extent of protection is similar to that observed in the DR6, Caspase-3, and Caspase-6 knockouts (Nikolaev et al., 2009; Simon et al., 2012), consistent with the model that APP, DR6, and the caspases function in a common pruning pathway.

These results provide the first direct evidence for a role of APP in axonal pruning in vivo. Our analysis also supports a cellautonomous role for both APP and DR6 in RGCs themselves (rather than the collicular target), because use of shRNAs to downregulate their expression in retinal cells in utero impairs pruning of RGC axons in the target. Importantly, our data also support the model that APP and DR6 function in the same pathway, because the effect on pruning of loss of function of APP and DR6 together was not greater than the effect of loss of DR6 alone.

The coincident phenotypes of APP and DR6 loss of function observed in retinotectal pruning also extend prior data on the neuromuscular junction, in which overshooting of motor axons was observed in mice lacking DR6 (Nikolaev et al., 2009), a phenotype also reported in mice double mutant for $A P P$ and its relative, APLP2 (Wang et al., 2005). Coincident loss-of-function phenotypes have also been observed for dendritic spines in cortical layers $2 / 3$, because loss of either APP or DR6 causes an increase in spine density that is dose dependent for both genes (i.e., loss of one APP or DR6 allele gives a partial phenotype that is increased further when the second allele of the gene is lost; Bittner et al., 2009; Kallop et al., 2014); furthermore, loss of both APP and DR6 (in APP;DR6 double-knock-out mouse) does not increase spine density beyond what is observed in either single mutant alone (Kallop et al., 2014) — a nonadditivity of effects that is again consistent with APP and DR6 functioning in the same pathway.

Collectively, these data support involvement of APP and DR6 in a common pathway that regulates diverse biological processes, including axonal pruning.

\section{Mechanism of APP and DR6 in pruning}

How do APP and DR6 control pruning? Prior results relying on pharmacological analysis in a model of sensory axon degeneration in vitro in response to trophic deprivation had suggested a model in which $\beta$-secretase cleavage of APP was followed by another cleavage, resulting in release of an N-terminal fragment of APP that bound DR6 to trigger degeneration (Nikolaev et al., 2009). We revisited this model because of our finding that pruning of the retinocollicular projection in vivo was not impaired in Bace-1 mutant mice.

To test the involvement of the various players in more detail, we studied degeneration in vitro of neurons isolated from mice carrying loss-of-function mutations in Bace-1, Bace-2, DR6, and $A P P$. This analysis unexpectedly revealed that several pharmacological reagents (DR6 and APP antibodies, small-molecule BACE inhibitors, and partially purified N-APP protein) used in prior analysis had off-target effects that affected the conclusions. Nonetheless, genetic analysis supports the model that DR6 and APP contribute to sensory axon degeneration following trophic deprivation in vitro. The protective effects of genetic deletion of $D R 6$ and $A P P$ are clear, with partial protection against degeneration observed $36 \mathrm{~h}$ after trophic deprivation. However, those protective effects are more modest than was previous observed with antibodies (Nikolaev et al., 2009), which we found reflects off-target effects of those reagents. They are also considerably more modest than the complete protection afforded by genetic deletion of Caspase-3 or Bax, which can be observed even $72 \mathrm{~h}$ after trophic deprivation (Nikolaev et al., 2009; Simon et al., 2012). This result indicates the operation of additional mechanisms that collaborate with APP and DR6 in pruning in vitro, consistent with partial protection seen in vivo for RGC axon pruning in DR6 and APP knock-out mice.

The analysis also showed that $\beta$-secretase activity is not required for pruning in vitro, because no protection was observed in cultures of neurons from Bace-1 knock-out or Bace-1;Bace-2 double knock-out mice (the protective action of pharmacological inhibitors was shown to reflect off-target actions). These data argue against $\beta$-secretase cleavage of APP being required for APP and DR6 function in vitro, which is consistent with the in vivo results in retinal ganglion cells. We also revisited the idea that an $\mathrm{N}$-terminal fragment of APP, proposed to be dependent on $\beta$-secretase, mediates high-affinity interaction of APP and DR6. This conclusion was based on use of partially purified N-terminal fragment (Nikolaev et al., 2009), but we found that the interaction was lost when it was fully purified, suggesting that the previously observed binding reflected presence of aggregates or other contaminants. In fact, further biochemical and more detailed structure-function analysis indicates that the high-affinity biochemical interaction of the full APP and DR6 ectodomains consistently observed in cell-based binding assays actually requires a portion of the APP ectodomain that is more C-terminal than previous appreciated (C-APP), residing in its so-called E2 domain; however, more $\mathrm{N}$-terminal sequences could also potentially contribute, because the affinity of binding of C-APP was lower than that of the full APP ectodomain (Fig. 7).

The picture that emerges from this analysis is that APP and DR6 are required for degeneration in both the in vitro and the in vivo paradigms studied here, but that $\beta$-secretase activity is not. Because APP can be cleaved by other proteases, we cannot exclude that APP cleavage is required for degeneration. However, our results also open the possibility that APP cleavage is not required, and that uncleaved APP could be the molecular species that regulates degeneration. If so, it is interesting to consider that cleavage of APP could potentially have the opposite effect, that is, to alleviate rather than enhance its prodegenerative effect-a finding that could explain our observation that retinal axon pruning in vivo was actually enhanced in Bace- 1 knock-out mice 
(Fig. 5). It is interesting that DR6 is also a $\beta$-secretase substrate (Hemming et al., 2009), so cleavage of DR6 could similarly reduce its prodegenerative actions, again potentially explaining why pruning was enhanced in the Bace-1 knock-out.

Given the high-affinity biochemical interaction between APP and DR6 ectodomains and the fact that they function in the same pathway, it is reasonable to assume that their direct physical interaction contributes to their actions. That possibility is nonetheless potentially consistent with multiple models, especially if the active species are uncleaved APP and DR6. For example, APP could function as a ligand for DR6 in cis in the cell membrane to trigger degeneration. Alternatively, the interaction could occur in reverse, with DR6 being a ligand that binds APP, which acts as a receptor to trigger degeneration. It is also possible that APP and DR6 form a coreceptor complex that responds to a separate ligand or activation mechanism. The fact that we did not observe prodegenerative effects of the soluble APP ectodomain or its fragments when fully purified (see Results) might be taken to provide support for the latter possibility. However, if uncleaved APP is normally the molecular species that regulates pruning, it is also possible that soluble fragments might not be effective in activation unless they are clustered in higher-order complexes than those tested here (as observed, for example, for activation of Eph receptors by soluble forms of their membrane-tethered ligands, which only activate their cognate receptors when highly clustered; Schaupp et al., 2014). Finally, even though APP and DR6 function in the same pathway, we cannot exclude that their highaffinity biochemical interaction might not be necessary for their function in pruning. Further studies are thus required to distinguish these and other possible models for how APP and DR6 contribute to axon degeneration and to determine the full extent of APP and DR6 involvement in developmental axon pruning throughout the nervous system.

\section{References}

Bittner T, Fuhrmann M, Burgold S, Jung CK, Volbracht C, Steiner H, Mitteregger G, Kretzschmar HA, Haass C, Herms J (2009) Gammasecretase inhibition reduces spine density in vivo via an amyloid precursor protein-dependent pathway. J Neurosci 29:10405-10409. CrossRef Medline

Chen M, Maloney JA, Kallop DY, Atwal JK, Tam SJ, Baer K, Kissel H, Kaminker JS, Lewcock JW, Weimer RM, Watts RJ (2012) Spatially coordinated kinase signaling regulates local axon degeneration. J Neurosci 32: 13439-13453. CrossRef Medline

Garcia-Frigola C, Carreres MI, Vegar C, Herrera E (2007) Gene delivery into mouse retinal ganglion cells by in utero electroporation. BMC Dev Biol 7:103. CrossRef Medline
Gray NW, Weimer RM, Bureau I, Svoboda K (2006) Rapid redistribution of synaptic PSD-95 in the neocortex in vivo. PLoS Biol 4:e370. CrossRef Medline

Guo Q, Li H, Gaddam SS, Justice NJ, Robertson CS, Zheng H (2012) Amyloid precursor protein revisited: neuron-specific expression and highly stable nature of soluble derivatives. J Biol Chem 287:2437-2445. CrossRef Medline

Hemming ML, Elias JE, Gygi SP, Selkoe DJ (2009) Identification of betasecretase (BACE1) substrates using quantitative proteomics. PLoS One 4:e8477. CrossRef Medline

Kallop DY, Meilandt WJ, Gogineni A, Easley-Neal C, Wu T, Jubb AM, Yaylaoglu M, Shamloo M, Tessier Lavigne M, Scearce-Levie K, Weimer RM (2014) A death receptor 6-amyloid precursor protein pathway regulates synapse density in the mature CNS but does not contribute to Alzheimer's disease-related pathophysiology in murine models. J Neurosci 34:6425-6437.

Luo L, O'Leary DD (2005) Axon retraction and degeneration in development and disease. Annu Rev Neurosci 28:127-156. CrossRef Medline

Marik SA, Olsen O, Tessier-Lavigne M, Gilbert CD (2013) Death Receptor 6 regulates adult experience-dependent cortical plasticity. J Neurosci 33: 14998-15003. CrossRef Medline

Nikolaev A, McLaughlin T, O'Leary DD, Tessier-Lavigne M (2009) APP binds DR6 to trigger axon pruning and neuron death via distinct caspases. Nature 457:981-989. CrossRef Medline

Schaupp A, Sabet O, Dudanova I, Ponserre M, Bastiaens P, Klein R (2014) The composition of EphB2 clusters determines the strength in the cellular repulsion response. J Cell Biol 204:409-422. CrossRef Medline

Schoenmann Z, Assa-Kunik E, Tiomny S, Minis A, Haklai-Topper L, Arama E, Yaron A (2010) Axonal degeneration is regulated by the apoptotic machinery or a NAD+-sensitive pathway in insects and mammals. J Neurosci 30:6375-6386. CrossRef Medline

Shan ZX, Lin QX, Yang M, Deng CY, Kuang SJ, Zhou ZL, Xiao DZ, Liu XY, Lin SG, Yu XY (2009) A quick and efficient approach for gene silencing by using triple putative microRNA-based short hairpin RNAs. Mol Cell Biochem 323:81-89. CrossRef Medline

Shaner NC, Campbell RE, Steinbach PA, Giepmans BN, Palmer AE, Tsien RY (2004) Improved monomeric red, orange and yellow fluorescent proteins derived from Discosoma sp. red fluorescent protein. Nat Biotechnol 22:1567-1572. CrossRef Medline

Simon DJ, Weimer RM, McLaughlin T, Kallop D, Stanger K, Yang J, O’Leary DD, Hannoush RN, Tessier-Lavigne M (2012) A caspase cascade regulating developmental axon degeneration. J Neurosci 32:17540-17553. CrossRef Medline

Vohra BP, Sasaki Y, Miller BR, Chang J, DiAntonio A, Milbrandt J (2010) Amyloid precursor protein cleavage-dependent and -independent axonal degeneration programs share a common nicotinamide mononucleotide adenylyltransferase 1-sensitive pathway. J Neurosci 30:13729-13738. CrossRef Medline

Wang P, Yang G, Mosier DR, Chang P, Zaidi T, Gong YD, Zhao NM, Dominguez B, Lee KF, Gan WB, Zheng H (2005) Defective neuromuscular synapses in mice lacking amyloid precursor protein (APP) and APP-Like protein 2. J Neurosci 25:1219-1225. CrossRef Medline 